\title{
Development of digital gamma-activation autoradiography for analysis of samples of large area
}

\author{
By V. P. Kolotov, D. S. Grozdov, N. N. Dogadkin and V. I. Korobkov* \\ Vernadsky Institute of Geochemistry and Analytical Chemistry of Russian Academy of Sciences, Moscow, Russia
}

(Received December 22, 2009; accepted in revised form December 20, 2010)

\begin{abstract}
Digital autoradiography / Gamma-activation /
Large samples / Dose equalization /

Acquired dose dynamics
\end{abstract}

\begin{abstract}
Summary. Gamma-activation autoradiography is a prospective method for screening detection of inclusions of precious metals in geochemical samples. Its characteristics allow analysis of thin sections of large size (tens of $\mathrm{cm}^{2}$ ), that favourably distinguishes it among the other methods for local analysis. At the same time, the activating field of the accelerator bremsstrahlung, displays a sharp intensity decrease relative to the distance along the axis. A method for activation dose "equalization" during irradiation of the large size thin sections has been developed. The method is based on the usage of a hardware-software system. This includes a device for moving the sample during the irradiation, a program for computer modelling of the acquired activating dose for the chosen kinematics of the sample movement and a program for pixel-by pixel correction of the autoradiographic images. For detection of inclusions of precious metals, a method for analysis of the acquired dose dynamics during sample decay has been developed. The method is based on the software processing pixel by pixel a time-series of coaxial autoradiographic images and generation of the secondary meta-images allowing interpretation regarding the presence of interesting inclusions based on half-lives. The method is tested for analysis of copper-nickel polymetallic ores. The developed solutions considerably expand the possible applications of digital gamma-activation autoradiography.
\end{abstract}

\section{Introduction}

Radioanalytical methods based on registration of images have a long history. In the last 60-70 years, these methods have played, perhaps, a leading role for local analysis. Autoradiography has been used to investigate the distribution either natural radionuclides, or artificial radionuclides induced from elements after sample activation (for example, by neutron irradiation). The last method has been intensively used for studying the spatial distribution of various elements in geological, cosmic and environmental samples and some technological materials. Over time, the appearance of powerful instrumental methods for local analysis (SEM,

\footnotetext{
*Author for correspondence (E-mail: kolotov@geokhi.ru)
}

$\mathrm{X}$-ray microanalysis, etc.) have resulted in a noticeable decrease of the role of radioanalytical methods including nuclear imaging ones. However, the potential of these methods is not still exhausted. Autoradiographic methods are in use now for research related to the safe immobilization of radionuclides [1,2], hot particles analysis $[3,4]$ investigation of dynamics of distribution of the labelled compounds in biological systems (plants, animals) [5] including in "on line" ("in vivo") variant [6]. Applications of PET with special laboratory equipment $[7,8]$ also may be considered as variant of digital autoradiography.

Earlier it has been shown that digital gamma-activation autoradiography provides effective detection of micro inclusions of noble elements in thin sections $[9,10]$. The native peculiarity of autoradiography is the possibility for analysis of large size samples (the areas of the available detectors reach dozens of $\mathrm{cm}^{2}$ ). It may conveniently be applied to screening analysis of large thin sections for searching "zones of interest" after which detailed analysis can be performed by means of a suitable instrumental method. It is necessary to take into consideration that usually electron accelerator bremsstrahlung has a sharp intensity decreasing away from axis. Therefore the activation of thin sections of large size $\left(\sim 20-30 \mathrm{~cm}^{2}\right)$ results in unacceptably high non-uniformity of the induced radioactivity over the sample surface. A solution for this problem is one of the tasks undertaken in this investigation.

On the other hand, screening analysis implies that results should be obtained as soon as possible. Therefore, it is highly desirable to do it as an automated variant. Consequently, the development of a specialized computer program for processing autoradiographic images is needed. As it is known, activation beta-autoradiography is based on integral recording of the interaction of radiation with detector (in the form of optical density that is proportional to acquired dose). To increase the selectivity of the method and consequently to increase the selectivity of the micro inclusions to be detected, it is proposed that pixel by pixel mathematical analysis of a time-series of images is performed while the activated sample cools (decays). The obtained data may be represented as derivative images (we called them metaimages), which contain information on the dynamics of the radioactivity decay for each pixel. At the beginning of the work it was not clear whether it was possible to recover halflife information from the optical densitometry data. 


\section{Experimental}

\subsection{Sampling, irradiation, gamma-ray spectrometry}

Samples for local analysis represent carefully polished thin sections (usually 1-2 mm of thickness) to ensure the highest spatial resolution. The samples were activated by microtron bremsstrahlung $\left(E_{\max }=25 \mathrm{MeV}\right)$ at the Institute of Metallurgy of Russian Academy of Sciences. The electron's current was $4-8 \mu \mathrm{A}$. The typical irradiation time was $30 \mathrm{~min}$. Gamma-ray spectra were measured by means of a HPGe detector (18\% of relative efficiency, $1.8 \mathrm{keV}$ resolution for $1332 \mathrm{keV}$ ) and a multichannel analyzer (both Canberra Inc.).

\subsection{Autoradiography}

BioMax MR nuclear film (Kodak) and recommended chemicals for photo processing were used for autoradiography. The obtained autoradiography images were converted to digital form by means of FilmScan 3600 slide scanner (Microtek) having a resolution of $3600 \mathrm{dpi}$ and a dynamic range of 3.0 D. The standard film Q-60 (Kodak) has been used for optical density calibration. The duration of autoradiogram acquisition covers the interval 0.5 to $26 \mathrm{~h}$ to obtain images with good balanced optical densities with maximum not exceeding 1 to $1.5 \mathrm{D}$ [11]. The treatment of the images has been done both by custom software made at the lab and Adobe Photoshop.

\section{Results and discussion}

\subsection{Equalization of the activation dose over the large size thin section surface}

To develop a system for the uniform irradiation of large size thin sections by bremsstrahlung, the following investigations were carried out: monitoring of topology of the activating field with the use of foils or artificial samples simulating thin sections; developing a device for movement of the sample at the irradiation site according to the chosen kinematic scheme; computer modelling of the activating dose acquisition during irradiation; and development of software and a method and for pixel-by-pixel correction of autoradiographic images for final "equalization" of the acquired activation dose over the image surface. It is worth noting that the $\mathrm{C} \#$ programming language is sufficiently powerful for any image processing. It is this language that was used for software development.

For monitoring the distribution of the bremsstrahlung, a disk-monitor was irradiated. The disk-monitor of $5 \mathrm{~cm}$ in diameter was made from calcium containing epoxy resin (Henkel), solidified for one day and polished. The irradiation was made at $9 \mathrm{~cm}$ distance from the tungsten converter for 30 min and with an electron current $\sim 5 \mu \mathrm{A}$. Dependence of the detector response on distance relative to the field axis is presented in Fig. 1. One can see that the activating field is represented by a narrow normal distribution. It is obvious, that the uniform irradiation of thin sections of large size (up to $50 \mathrm{~mm}$ in diameter) is impossible. A device which would provide movement of the sample in the plane perpendicular to the field axis is required. The device should be simple enough to ensure its reliable functioning in a high-intensity bremsstrahlung.

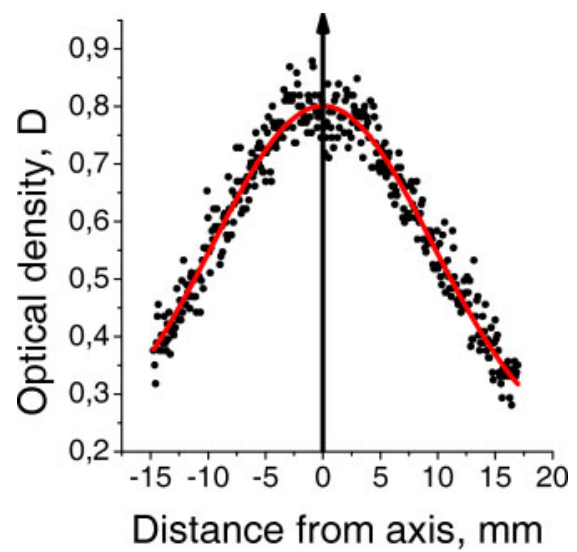

Fig. 1. Distribution of the relative intensity of microtron bremsstrahlung.

The suggested kinematic scheme of moving large size thin sections for uniform irradiation is presented in Fig. 2. The device makes simultaneous the rotation and alternate/ reciprocal motion of the platform with the fixed thin section(s). Such a scheme does not demand the development of a sophisticated controller, because the parameters of the activating field distribution, possible drift of its centre, etc. may be not taken into account (in contrast, for example, to scanning movement).

Computer 2D-modelling of activating dose acquisition (program DoseModelling) has preceded the assemblage and test of the pilot device for irradiation. It is necessary to conduct this computation to estimate the uniformity of the dose at various parameters of the device movement. The acquired dose was computed by means of integration of the "projected" 2D-activating field on the square matrix of $50 \times 50$ elements, where each element approximately corresponded to the area of $1 \mathrm{~mm}^{2}$. In the Cartesian system of co-ordinates the position of the centre of the activating field depending on the passed time from the irradiation beginning and the selected kinematics of movement can be given by the following equations (1)-(2):

$$
\begin{aligned}
& x_{c}=R_{1}+R_{2} \cos \left(\omega_{2} t\right) \cos \left(\omega_{1} t\right) \\
& y_{c}=R_{1}+R_{2} \cos \left(\omega_{2} t\right) \sin \left(\omega_{1} t\right)
\end{aligned}
$$

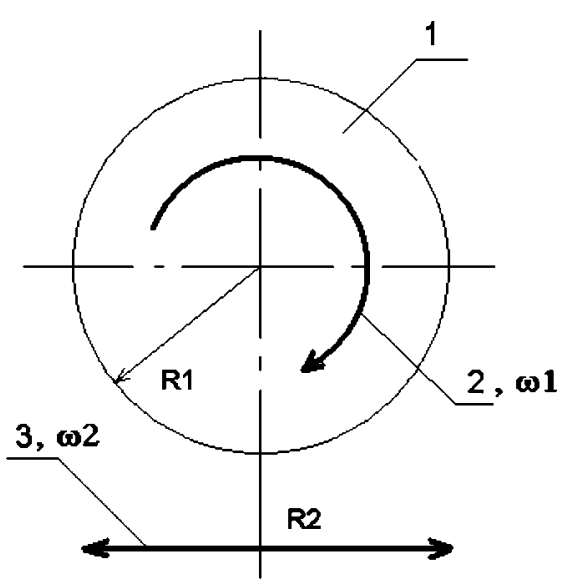

Fig. 2. Kinematic scheme of the sample movement: 1 - sample with radius $=R_{1} ; 2$ - sample rotation with frequency $\omega_{1} ; 3-$ alternate/reciprocal motion with frequency $\omega_{2}$ and amplitude $R_{2}=R_{1}$. 2 . 


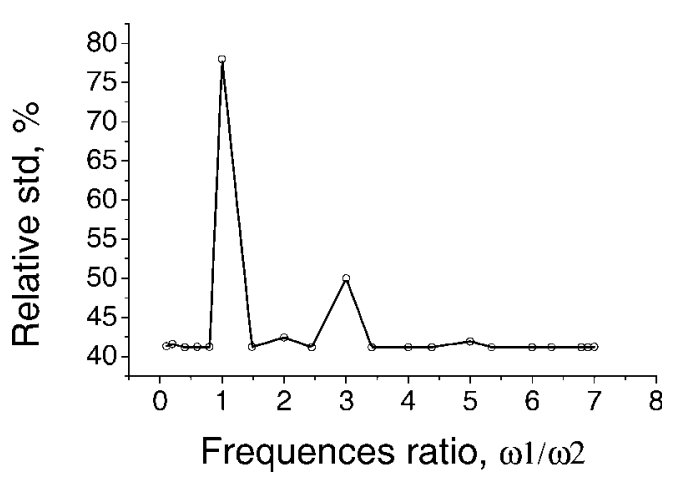

Fig. 3. Dependence of the relative standard deviation of the acquired dose on the sample motion frequencies ratio.

where: $R_{1}$ - radius of the sample, $2 \times R_{2}$ - amplitude of horizontal movement, $\omega_{1}$ - frequency of rotation, $\omega_{2}-$ frequency of alternate/reciprocal motion, $t$ - time passed since the start of irradiation. The centre of the sample has coordinates: $x=R_{1}, y=R_{1}$.

For estimation of the integral dose both a real normal distribution and a rectangular distribution for the activating radiation has been used. The time of the irradiation during the modelling experiment has been divided into intervals (so-called "quants of time") used for dose integration.
The program DoseModelling requests initial parameters of the device movement, irradiation duration, type and parameters of distribution of bremsstrahlung. As a measure of uniformity of the acquired dose a relative standard deviation of the integrated dose over the whole sample surface (a circle of radius R1) has been used. For some ratios of frequencies of rotary and alternate/reciprocal motion some irregularities (resonances) of nonuniformity of the integrated dose are observed (Fig. 3). This effect is caused by absence of irradiation of a part of thin section that is illustrated by Fig. 4.

In Fig. 5, the distribution of the acquired dose over the surface of the sample $(d=50 \mathrm{~mm})$ is presented. The cross section through the surface of the acquired dose is presented in Fig. 6. One can see that the device provides considerable alignment of a dose, especially in peripheral parts of the sample, where in case of the motionless sample the acquired dose is practically zero. At the same time, residual nonuniformity of the dose is still preserved. This non-uniformity has either normal distribution relative to the axis of radiation, or may be represented by the sum of two identical normal distributions. The latter case means that sometimes it is not possible to install the centre of the sample rotation strictly in alignment with the activating field axis. In this case the distance between centres is a doubled displacement
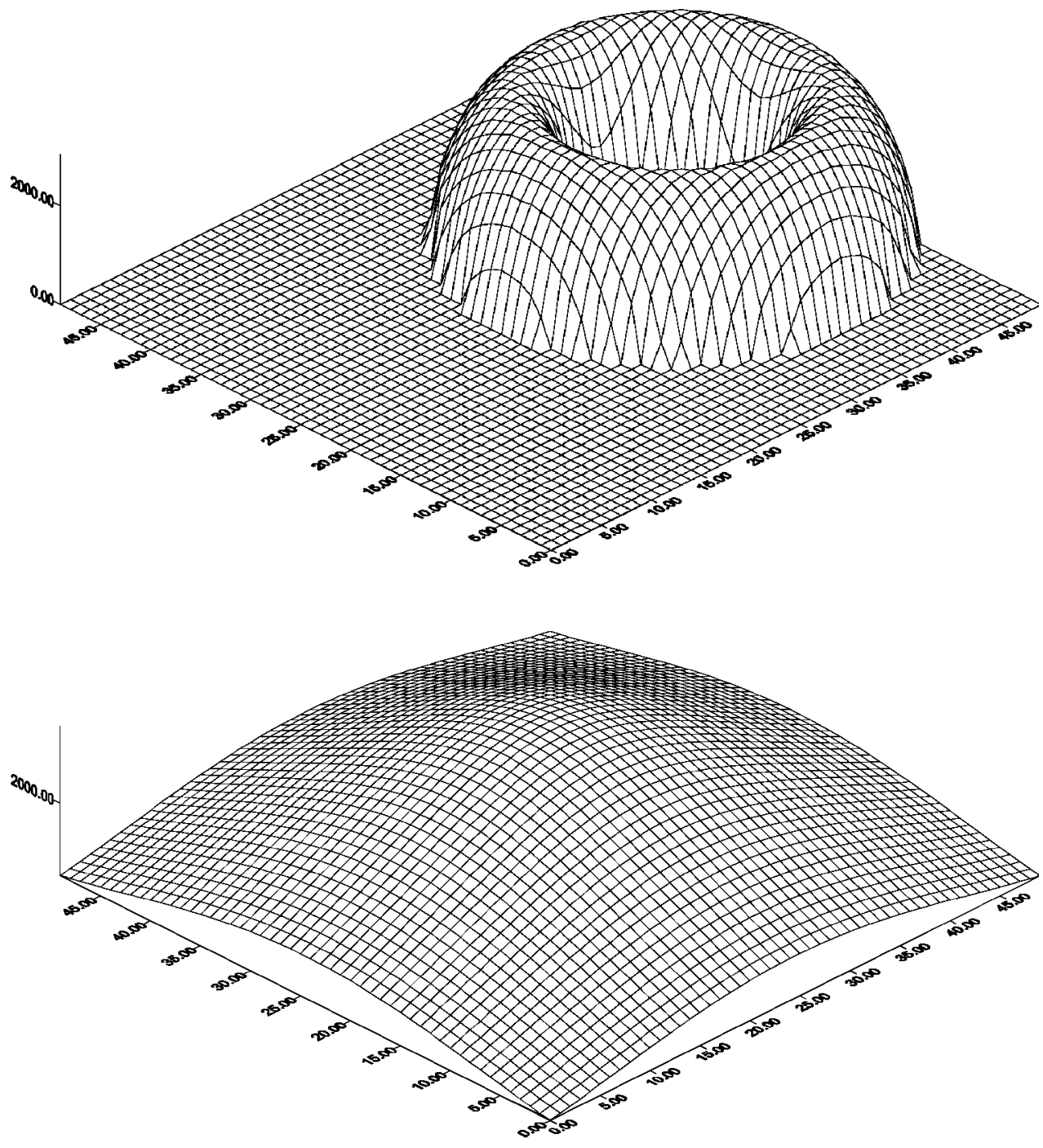

Fig. 4. 2D distribution of the acquired dose over the sample surface using the conditions of movement corresponding the first resonance $\left(\omega_{1} / \omega_{2}=1\right.$, Fig. 3$)$ and rectangular distribution of the activation field (to increase contrast)
Fig. 5. 2D-distribution of the acquired dose over the sample using the real distribution of the activation field $\left(\omega_{1} / \omega_{2}=6.9\right)$. 


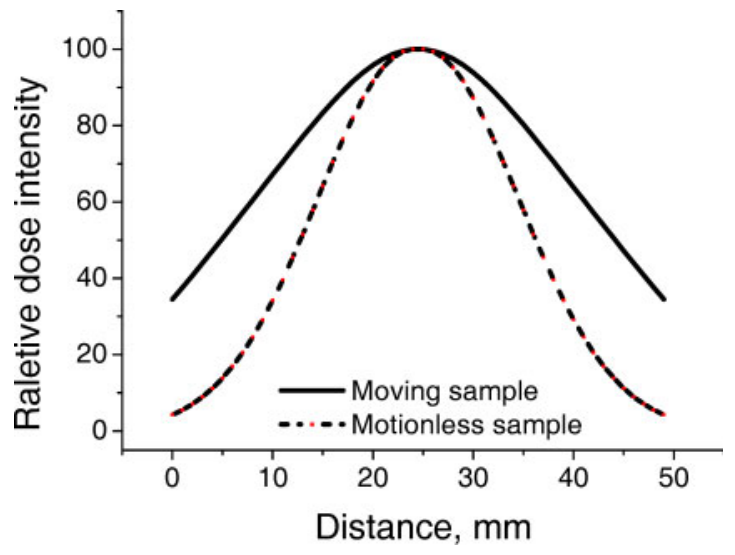

Fig. 6. Distribution of the acquired dose over the sample for a moving and a motionless sample.

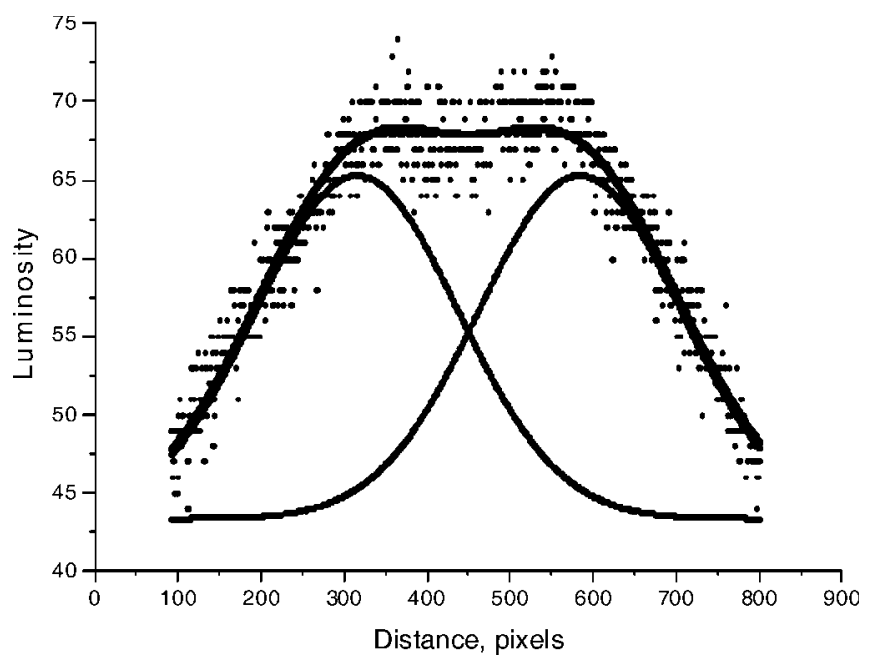

Fig. 7. Dose acquired by the copper monitor. Two similar distributions mean that sample rotation and bremstrahlung are not coaxial.

of axes (Fig. 7) for sample rotation and bremstrahlung. In any case, the residual non-uniformity of the acquired dose can be corrected mathematically.
For the control of conditions of the irradiation it is suggested that an activating field monitor is used (a copper foil disk fixed to the back side of the sample). The monitor has a system of labels (apertures) for the subsequent coaxial positioning of autoradiographic images of the sample and the monitor. The resulting autoradiograms are analyzed to reveal the of alignment of the sample rotation relative to the axis of the activating field.

The obtained parameters are used by the developed program DoseCorrection. This program makes pixel by pixel correction of autoradiographic image for the final equalization of the acquired dose. The correction consists in computation of the normalising factor for each pixel of the image relying on the developed algorithm. After correction the program stores the new image on a hard disk for the subsequent quantitative analysis of the autoradiograms.

On the basis of the accepted kinematic scheme the device for uniform irradiation of the samples has been developed and tested. The devise uses two low-speed engines (with possibility of adjustment of number of turns) for rotary and alternate/reciprocal motion.

\subsection{Processing of a series of autoradiography images}

The primary goal of activation beta autoradiography is the choice of the irradiation/cooling conditions for reaching the best selectivity of the target radionuclide(s) registration. The task necessitates obtaining the of most contrast of zones with high concentration of analytical radionuclide relative to the activated matrix. The activation autoradiography is not a selective method, however, application of computer processing of a series of the autoradiograms obtained in the course of decay of rather short lived radionuclides allows changing the situation essentially. The developed computer processing consists of following stages: positioning of a series of images in coaxial layers; pixel by pixel data processing for estimation of dynamics of optical density change in time (estimation of rate of decay); interpretation and representation of the obtained results. In other words, with the use

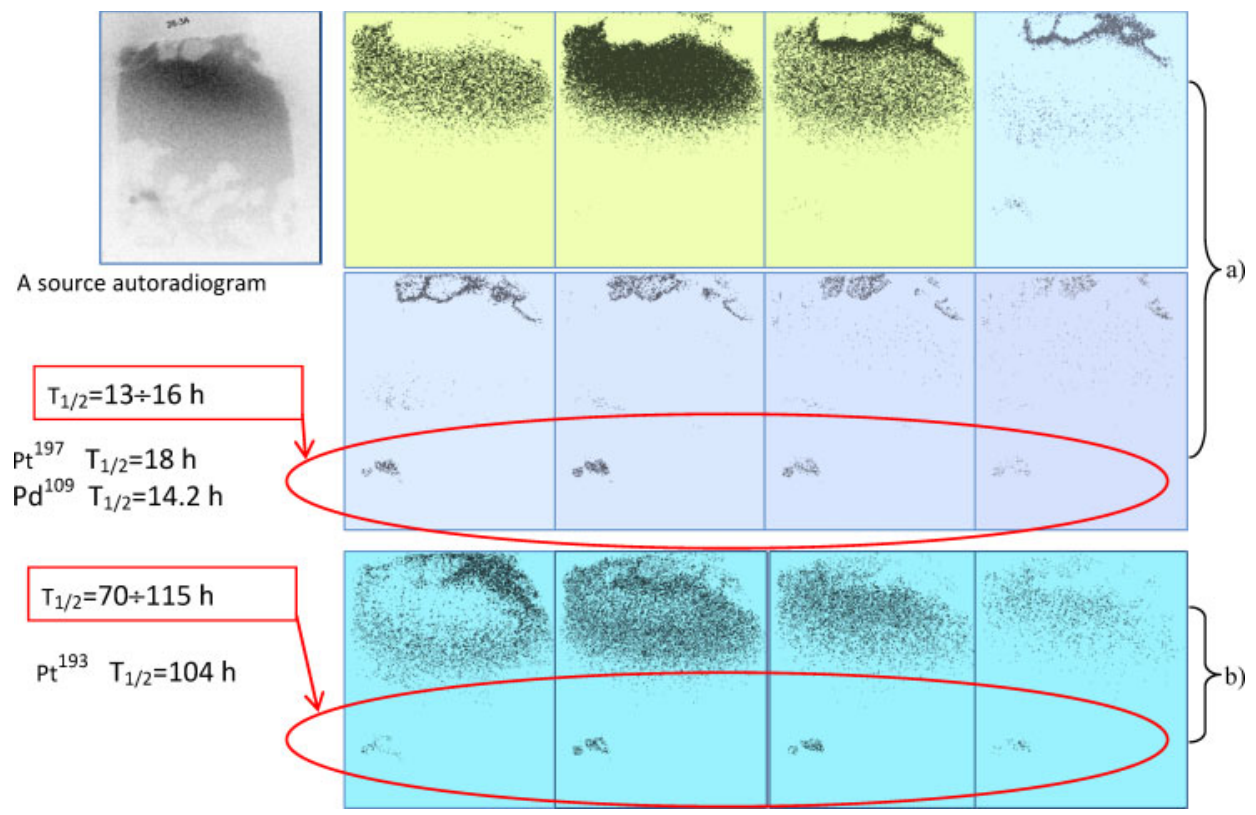

Fig. 8. A selected autoradiogram from a processed time-series (upper-left) and a set of metaimages for different half-life intervals (ellipse indicates zone of Pd-Pt inclusion). (a) Short lived radionuclides: $9-16 \mathrm{~h}(1 \mathrm{~h}$ increment), (b) long lived radionuclides: $70-115 \mathrm{~h}$ (15 h increment). 
of data sets received from coaxially located pixels of all images of a series, the components half-life composing radionuclides mix should be estimated for each pixel of the image. Further, on the basis of the received results a series of the derivative meta-images, with information on the dynamics of decay may be generated. For transformation of luminosity values of the image pixels $(L=0 \div 255)$ to optical density a corresponding calibrations have been used [11]. Now the program is capable to process a mix containing maximum two radionuclides. This limitation is related with reproducibility of optical density detection and number of autoradiograms to be acquired. The ratio between half lifes for short lived and long lived detectable component usually is more than 4 . The program provides estimation of residuals for each pixel while approximation. The array of residuals forms the correspondent meta-image. The received information is curious for researches because it reveals the features of distribution of various elements (or their mixtures) and permits fast detection of the inclusions containing platinum group elements.

As an example, in Fig. 8, the autoradiograms of a processed series is given along with a number of the derivative meta-images corresponding to various half-lifes. The results refer to the samples of polymetallic ores containing high concentrations of copper and nickel. The received metaimages allow detection of zones corresponding to inclusions of platinum-palladium (Fig. 8). Presence of two Pd-Pt inclusions have been confirmed by SEM analysis.

The research has shown that pixel by pixel mathematical analysis of a time-series of images for an estimation of dynamics of radionuclides decay is rather informative even for images having standard 8 bit depth of grey. Future progress of the method will involve development of steady computational algorithms and a methodology for the interpretation of the received data.

Such analysis is applicable both for autoradiograms received by means of traditional photographic detectors and for the autoradiograms received by means of direct digital technologies (for example, by Fuji-Film's Image Plates).

\section{Conclusion}

The developed system for irradiation of large size thin sections and the processing of an autoradiographic time-series of images significantly extends the capabilities of digital gamma-activation analysis for screening local analysis of geological samples. It has been demonstrated that computer processing of the autoradiogram time-series obtains information on decay dynamics for each pixel of the image even having 8 bits depth. Such analysis may be considered as the first step for making mapping of elements.

Acknowledgment. The work is supported by the Russian Fund for Basic Research (grant N 10-03-00140-a). We express our thanks to V. I. Tovtin and V. M. Lazorenko (both IMET RAS) for help during irradiation of the samples.

\section{References}

1. Vinokurov, S. E., Kulyako, Yu. M., Slyuntchev, O. M., Rovny, S. I., Myasoedov, B. F.: Low-temperature immobilization of actinides and other components of high-level waste in magnesium potassium phosphate matrices. J. Nucl. Mater. 385, 189 (2009).

2. Vinokurov, S. E., Kulyako, Yu. M., Slyuntchev, O. M., Rovny, S. I., Wagh, A. S., Maloney, M. D., Myasoedov, B. F.: Magnesium potassium phosphate matrices for immobilization of high-level liquid wastes. Radiochemistry 51(1), 65 (2009).

3. Zeissler, C. J., Lindstrom, R. M., McKinley, J. P.: Radioactive particle analysis by digital autoradiography. J. Radioanal. Nucl. Chem. 248(2), 407 (2001).

4. Kerkapoly, A., Vajda, N., Pinter, T.: Film autoradiography used for hot particle identification. J. Radioanal. Nucl. Chem. 265(3), 423 (2009).

5. Badun, G. A., Kulikova, N. A., Chernysheva, M. G., Tyasto, Z. A., Korobkov, V. I., Fedoseev, V. M., Tsvetkova, E. A., Konstantinov, A. I., Kudryavtsev, A. V., Perminova, I. V.: Tritium labeling: a unique tool for studying the behavior of humic substances in living systems. Moscow Univ. Chem. Bull. 64(5), 276 (2009).

6. Kanno, S., Rai, H., Ohya, T., Hayashi, Y., Tanoi, K., Nakanishi, T. M.: Real-time imaging of radioisotope labeled compounds in a living plant. J. Radioanal. Nucl. Chem. 272(3), 565 (2007).

7. Furukawa, J., Yokota, H., Tanoi, T., Ueoka, S., Matsuhashi, S., Ishioka, N. S., Watanabe, S., Uchida, H., Tsuji, A., Ito, T., Mizuniwa, T., Osa, A., Sekine, T., Hashimoto, S., Nakanishi, T. M.: Vanadium uptake and an effect of vanadium treatment on ${ }^{18} \mathrm{~F}$ labeled water movement in a cowpea plant by positron emitting tracer imaging system (PETIS). J. Radioanal. Nucl. Chem. 249(2), 495 (2001).

8. Watanabe, S., Iida, Y., Suzui, N., Katabuchi, T., Ishii, S., Kawachi, N., Hanaoka, H., Watanabe, S., Matsuhashi, S., Endo, K., Ishioka, N. S.: Production of no-carrier-added ${ }^{64} \mathrm{Cu}$ and applications to molecular imaging by PET and PETIS as a biomedical tracer. J. Radioanal. Nucl. Chem. 280(1), 199 (2009).

9. Kolotov, V. P., Shilobreeva, S. N., Andriyanov, A. Yu., Dogadkin, N. N., Chapyzhnikov, B. A., Alenina, M. V.: Development of digital autoradiography technique for the determination of the platinum-group elements in geological materials. Radiochim. Acta 89, 765 (2001)

10. Kolotov, V. P., Dogadkin, N. N., Korobkov, V. I., Grozdov, D. S.: Determination of platinum-palladium micro inclusions in polymetallic ores by means of digital gamma-activation autoradiography. J. Radioanal. Nucl. Chem. 278(3), 739 (2008).

11. Kolotov, V. P., Andriyanov, A. Yu., Shilobreeva, S. N., Korobkov, V. I., Dogadkin, N. N., Chapyzhnikov, B. A., Tsipenyuk, Yu. M.: Development of digital gamma-activation autoradiography for the determination of platinum group elements inclusions in geological samples. J. Radioanal. Nucl. Chem. 271(3), 671 (2007). 